\title{
Plasmonic Bloch oscillations in chirped metal-dielectric structures
}

\author{
Artur R. Davoyan, ${ }^{a}$ Ilya V. Shadrivov, Andrey A. Sukhorukov, and Yuri S. Kivshar \\ Nonlinear Physics Center, Research School of Physics and Engineering, Australian National University, \\ Canberra, Australian Capital Territory 0200, Australia
}

(Received 26 November 2008; accepted 24 February 2009; published online 21 April 2009)

\begin{abstract}
We study the propagation of plasmon polaritons in one-dimensional chirped metal-dielectric layered structures. We find an optical Wannier-Stark ladder in the mode spectrum and analyze Bloch oscillations associated with the coupling of surface plasmons localized at the metal-dielectric interfaces. For long structures, we find that the energy flow may dramatically change its direction, thus providing possibilities for the beam steering in the transmission band. () 2009 American Institute of Physics. [DOI: 10.1063/1.3119666]
\end{abstract}

Recent technological advances opened up many opportunities for nanofabrication allowing one to study the fundamental effects earlier predicted only theoretically. As an example, electronic Bloch oscillations predicted in 1928 (Ref. 1) were observed almost 50 years later, ${ }^{2}$ when semiconductor superlattices with nanometer-scale thick layers were manufactured. Nowadays, one of the most intriguing directions of research is associated with plasmon-polariton excitations and light manipulation at nanoscales ${ }^{3,4}$ in various metal-dielectric structures, including multilayered periodic metal film stacks.

Periodic metal-dielectric stacks have been studied in a number of papers. ${ }^{5-7}$ The studies of the transmission properties ${ }^{6,7}$ revealed that such structures may exhibit resonant transmission due to plasmon tunneling through the structure. This effect by itself is quite remarkable, since every second layer in the structure is made of metal, i.e., it is opaque. It was suggested that these structures can be used as spatial filters. ${ }^{7}$

Optical Bloch oscillations in periodic dielectric structures represent an analog of the electronic Bloch oscillations in crystals. Such oscillations were observed in different dielectric structures, ${ }^{8-11}$ and they were also predicted to occur in metal-dielectric structures ${ }^{12,13}$ where it was shown that the structures with spatially modulated refractive index of dielectric layers can exhibit optical Bloch oscillations. ${ }^{12}$ More complex heterostructures containing coupled dielectric cavities sandwiched between the metal-dielectric waveguides were also shown to exhibit Bloch oscillations, ${ }^{13}$ somewhat similar to those observed in dielectric superlattices. ${ }^{11}$

In this letter we study the beam propagation in structures with linearly varying (chirped) thickness of the dielectric layers and uniform thickness of metallic layers. We predict the possibility of plasmonic Bloch oscillations arising due to the excitation and coupling of plasmon polaritons. We show that the energy flow can change its direction within one period of oscillations, leading to the beam curling similar to that predicted for layered structures with left-handed metamaterials. ${ }^{14}$ Such flexibility of beam control can open up opportunities of the light manipulation on nanoscales.

We start our analysis by studying periodic onedimensional stack consisting of thin metal layers of the width $h$ separated by layers of conventional dielectric of the width $l$, so that the period of the structure is defined as $\Lambda=h+l$. The

${ }^{a)}$ Electronic mail: ard124@rsphysse.anu.edu.au. refractive index in one unit-cell of the structure is written as

$$
n^{2}(x)= \begin{cases}n_{m}^{2}=\varepsilon_{m}<0 & x \in(0, h), \\ n_{d}^{2}=\varepsilon_{d}>0 & x \in(h, \Lambda),\end{cases}
$$

where $n_{m}$ and $n_{d}$ are the refractive indices of metal and dielectric, respectively.

We consider the propagation of TM-polarized monochromatic waves with the magnetic field vector $\mathbf{H}$ $=\left(0, H_{y}, 0\right)$, where $H_{y}(x, z)$ satisfies the Helmholtz equation,

$$
\Delta H_{y}(x, z)+n^{2}(x) H_{y}(x, z)-\frac{1}{\varepsilon} \frac{d \varepsilon(x)}{d x} \frac{\partial H_{y}(x, z)}{\partial x}=0,
$$

$\Delta$ is the two-dimensional Laplacian, and the spatial coordinates are normalized to $\lambda / 2 \pi$, where $\lambda$ is a wavelength in vacuum.

For a periodic structure, we apply the Floquet theorem ${ }^{15}$ and express the magnetic field as a superposition of forward and backward Bloch waves, $H_{y}(x, z)=\left[U_{+}(x) \exp \left(-j K_{B} x\right)\right.$ $\left.+U_{-}(x) \exp \left(j K_{B} x\right)\right] \exp \left(i k_{p} z\right)$, where the envelopes $U_{ \pm}$are periodic, $U_{ \pm}(x+\Lambda)=U_{ \pm}(x)$, and $K_{B}$ is the Bloch wave number satisfying the dispersion relation ${ }^{7,15}$

$$
\begin{aligned}
2 \cos \left(K_{B} \Lambda\right)= & 2 \cosh \left(\kappa_{m} h\right) \cosh \left(\kappa_{d} l\right) \\
& -\left(\alpha+\alpha^{-1}\right) \sinh \left(\kappa_{m} h\right) \sinh \left(\kappa_{d} l\right) .
\end{aligned}
$$

Here $\alpha=\kappa_{m} \varepsilon_{d} / \kappa_{d} \varepsilon_{m}, \kappa_{m, d}=\mp \sqrt{k_{p}^{2}-\varepsilon_{m, d}}$, and $k_{p}$ is the plasmon propagation constant normalized to $2 \pi / \lambda$.

Imaginary solutions for $K_{B}$ correspond to the waves decaying exponentially across the structure due to the presence of photonic bandgaps for corresponding range of $k_{p}$. Based on Eq. (3), we investigate the bandgap properties of the structure with fixed metal film thickness as a function of the dielectric layer width. We consider metal layers with permittivity $\varepsilon_{m}=-0.85$ and thickness $h=0.5$ (i.e., $\lambda / 4 \pi$ ) separated by vacuum, $\varepsilon_{d}=1$. As an example, we assume Ag films with $\varepsilon_{m}=-0.85$ for the wavelength around $400 \mathrm{~nm}$, and corresponding film thickness $h \sim 30 \mathrm{~nm}$. For simplicity we do not take into account the losses in metals. A corresponding bandgap diagram is shown in Fig. 1 on the parameter plane $\left(l, k_{p}\right)$.

Figure 1 (right) shows transmission bands due to plasmon-polariton tunneling for $k_{p}>\varepsilon_{d}$, where the waves are evanescent in both dielectric and metal. For smaller wave numbers, when $k_{p} \leq \varepsilon_{d}$, plasmon modes do not exist and the 


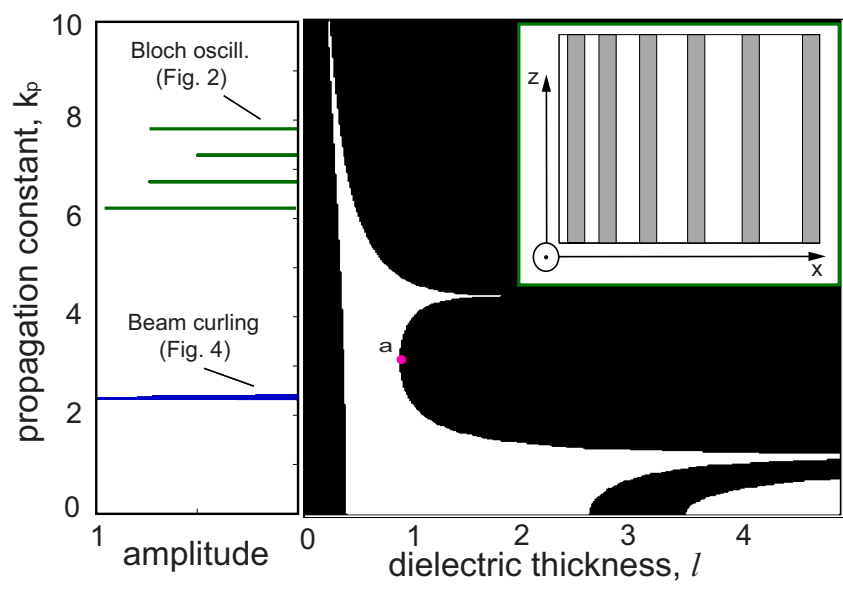

FIG. 1. (Color online) Right: Bandgap diagram for a one-dimensional structure consisting of metal films $\left(\varepsilon_{m}=-0.85, h=0.5\right)$ separated by vacuum. Black areas correspond to gaps, while white areas indicate bands. The inset shows a schematic of the chirped structure where shaded regions correspond to metallic layers with fixed thickness. Left: Spectra of eigenmodes for the chirped structure corresponding to the plasmonic Bloch oscillations (upper part of the spectra), and to the beam curling (lower part).

waves can propagate in dielectric layers. However, due to tunneling through thin metal layers, several transmission bands are formed in this regime too.

Now we introduce a linear chirp into the structure by changing the thickness of dielectric layers, $l_{N}=l_{0}+N \delta l$, where $N$ is unit cell number, and $\delta l$ is thickness gradient (see inset in Fig. 1). To study short structures with relatively small number of layers and large thickness gradient, we use the previously developed approach. ${ }^{16}$ In brief, we find eigenmodes of the structure by discretizing Eq. (2) and solve an eigenvalue problem of the emerging matrix. We analyze the spectrum of eigenvalues $k_{p}$ and find the regions where the wave numbers are equidistantly spaced. The equidistant eigenvalues $k_{p}$ correspond to a spatial optical equivalent of the Wannier-Stark ladder associated with the Bloch oscillations. ${ }^{8}$

We analyze the structure with 40 unit cells with thickness of dielectric layers changing from $l=0.2$ to $l=1.6$ so that the structure covers the transmission band including the adjacent gap layers [see Fig. 1 (right)]. For this structure we find an equidistant set of the propagation constants $k_{p}$ centered at $k_{p 0} \sim 7$, as shown in Fig. 1 (left). Exciting the corresponding modes by a Gaussian beam, we observe spatial beam oscillations shown in Fig. 2. We note that in our case

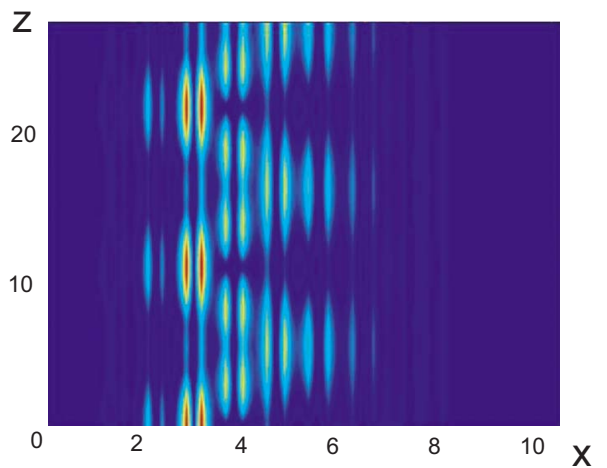

FIG. 2. (Color online) Evolution of the magnetic field amplitude demonstrating plasmonic Bloch oscillations in the structure with 40 unit cells. Oscillations period is $L_{z}=10.5$.

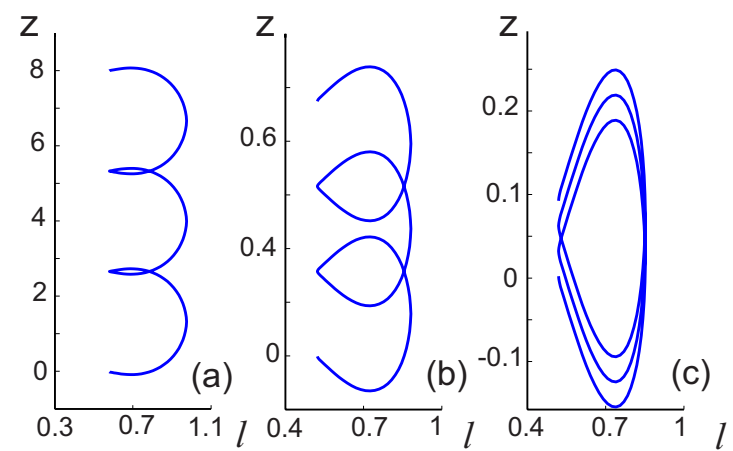

FIG. 3. (Color online) Trajectories of paraxial beams in the transmission band for different values of the propagation constants: (a) $k_{p}^{0}=1.7$, (b) $k_{p}^{0}$ $=2.3$, and (c) $k_{p}^{0}=2.5$.

of lossless materials, the beam completely reconstructs its shape after each period of oscillations. The plasmon field is highly confined to the metal interfaces, demonstrating that such Bloch oscillations appear solely due to the coupling between surface plasmons excited at different interfaces. The distance between the Wannier-Stark eigenstates $\Delta k_{p}$ defines the period of oscillations, $L_{z}=2 \pi / \Delta k_{p}$. For this particular case, we find $L_{z}=10.5$, and this value agrees with Fig. 2 .

Next we analyze long structures with a large number of layers. For adiabatically slow changing thickness of dielectric layers, $\delta l \rightarrow 0$, we use the geometric optics approximation and describe the evolution of paraxial beams. We introduce the ray trajectories along the averaged Poynting vector across the unit cell ${ }^{14}$

$$
\frac{\left\langle S_{z}\right\rangle}{\left\langle S_{x}\right\rangle}=-\left(\frac{\partial k_{p}}{\partial K_{B}}\right)^{-1} \equiv-\frac{1}{\nu},
$$

where $\left\langle S_{z, x}\right\rangle$ are the $z, x$ components of the averaged Poynting vector, respectively; $\nu$ is the spatial velocity of the ray, $\langle\cdot\rangle=\Lambda_{-1} \int_{\Lambda}(\cdot) d x$, which denotes averaging over the unit cell.

According to this equation, if $\partial k_{p} / \partial K_{B}$ changes its sign in the transmission band, the power flux $\left\langle S_{z}\right\rangle$ changes its direction, ${ }^{14}$ thus the beam injected into the structure may curl. This regime can be realized for the propagation constants $k_{p}^{0}$ close to the point where $\partial K_{B} / \partial k_{y}=0$. We find that this condition is satisfied at the boundary of transmission band near the point (a) in Fig. 1 (right).

Based on Eq. (4) we derive the ray motion equation

$$
\frac{d l}{d z}=\mp \frac{\partial k_{p}}{\partial K_{B}},
$$

that we employ to calculate the ray trajectories launched near the left edge of the structure for different $k_{p}$. For $k_{p}=1.7$ we observe the beam curling near left band boundary, as shown in Fig. 3(a). Further increase of the propagation constant leads to an increase of the curl size, and decrease of the spatial oscillation period [see Fig. 3(b)]. Finally, at $k_{p}^{0} \simeq 2.5$ the curl reaches the other transmission band edge forming practically closed trajectory with vanishing period of oscillations [see Fig. 3(c)]. Note that near the left edge, the energy flow in the $z$-direction is negative but remains positive near the right band edge. Further increasing the wave number results in the opposite process with the curl forming near the right band edge.

Finally, we reconstruct the field distribution in the regime of beam curling. Our previous approach based on the 


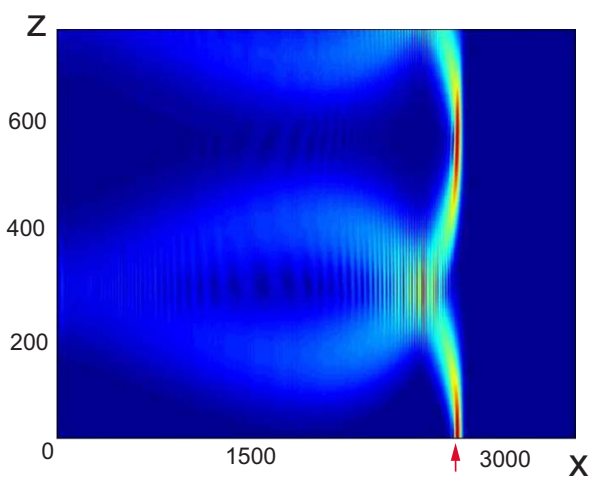

FIG. 4. (Color online) Evolution of the magnetic field demonstrating the beam curling for a Gaussian beam launched at right band boundary (shown by an arrow) with its spectrum centered at $k_{p}=2.3$.

discretization of the wave equation is computationally intensive, and it is not suitable for the present problem since the structure contains a larger number of layers. Therefore, we implement another approach based on the Bloch wave formalism. ${ }^{14}$ For the slowly varying period of the structure (i.e., $\delta l \ll l$ ) the field can be presented as a superposition of the forward- and backward-propagating Bloch waves with slowly varying amplitudes, $A_{\Lambda}$ and $B_{\Lambda}$.

We assume that the structure with a linearly varying dielectric layer thickness is placed between two semi-infinite periodic structures, with the period coinciding with that of the adjacent layers of the structure. In the left semi-infinite structure, the fields decay exponentially, and we choose the amplitudes in the first layer as $A_{\Lambda_{l}}=1$ and $B_{\Lambda_{l}}=0$. In the right semi-infinite structure the forward wave vanishes, and this means that $A_{\Lambda_{r}}=0$ and $B_{\Lambda_{r}}=1$. We calculate the amplitudes in the adjacent unit cells using the boundary conditions. Repeating this procedure, we find the amplitudes in the whole structure. Using this method, we find the spectrum of eigenvalues $k_{p}$ and corresponding eigenmodes of the structure. Then, the initial field distribution is represented as a superposition of the eigenmodes, and the complete field structure can be retrieved.

As an example, we calculate the eigenmodes for the structure consisting of 2500 unit cells with the width of vacuum layers changing linearly from the value $l_{l} \simeq 0.4$ to the value $l_{r} \simeq 1.2$, and $\delta l \simeq 2.8 \times 10^{-4}$. We note that for the wavelength $\lambda=400 \mathrm{~nm}$, average unit cell size is $\langle\Lambda\rangle$ $\simeq 80 \mathrm{~nm}$ and the structure width is about $200 \mu \mathrm{m}$. We assume that a Gaussian beam is launched near the right band boundary of the structure (see Fig. 4). The width of the beam is $a=1.3$, i.e., about 20 unit cells are exited, with the spectrum centered near $k_{p}^{0}=2.33$. We decompose the initial field distribution into the superposition of eigenmodes of the structure by using the least-squares method. The spectrum of the excited eigenmodes is shown in Fig. 1 (left). The spectrum is close to equidistant with spacing of $\delta k_{p}$, and accordingly the field restores its shape after the distance $L_{z}$ $=2 \pi / \delta k_{p}$. We plot the magnetic field amplitude averaged over the unit cell. The beam curling predicted in the framework of the geometric optics is clearly observed in the numerical simulations. At the beam self-crossing point, we observe an interference pattern created by the forward- and backward-propagating waves. The field distribution is restored almost completely after the first period of oscillations. Our simulations are in good agreement with geometric optics approximation [see Fig. 3(b)].

In conclusion, we have studied plasmonic Bloch oscillations in chirped metal-dielectric layered structures with a linearly varying thickness. We have described a type of Bloch oscillation and revealed the effect of the beam curling due to the backward energy flow. We expect that the predicted phenomena can be observed in the end-fire coupling geometry with the multilayer metal-dielectric structures, when the beam launched at a particular angle will reappear from the same facet of the structure with some offset.

The work has been supported by the Australian Research Council.

${ }^{1}$ F. Bloch, Z. Phys. 52, 555 (1928).

${ }^{2}$ C. Waschke, H. G. Roskos, R. Schwedler, K. Leo, H. Kurz, and K. Kohler, Phys. Rev. Lett. 70, 3319 (1993).

${ }^{3}$ V. M. Agranovich and D. L. Mills, Surface Polaritons (North-Holland, Amsterdam, 1982).

${ }^{4}$ S. A. Maier, Plasmonics: Fundamentals and Applications (Springer, Berlin, 2007).

${ }^{5}$ L. G. Wang, H. Chen, and S. Y. Zhu, Phys. Lett. A 350, 410 (2006).

${ }^{6}$ H. Jiang, H. Chen, H. Li, Y. Zhang, J. Zi, and S. Zhu, Phys. Rev. E 69, 066607 (2004).

${ }^{7}$ S. Feng, J. Elson, and P. Overfelt, Opt. Express 13, 4113 (2005).

${ }^{8}$ C. Martijn de Sterke, J. N. Bright, P. A. Krug, and T. E. Hammon, Phys. Rev. E 57, 2365 (1998).

${ }^{9}$ T. Pertsch, P. Dannberg, W. Elflein, A. Brauer, and F. Lederer, Phys. Rev. Lett. 83, 4752 (1999).

${ }^{10}$ R. Sapienza, P. Costantino, D. Wiersma, M. Ghulinyan, C. J. Oton, and L. Pavesi, Phys. Rev. Lett. 91, 263902 (2003).

${ }^{11}$ V. Agarwal, J. A. del Rio, G. Malpuech, M. Zamfirescu, A. Kavokin, D. Coquillat, D. Scalbert, M. Vladimirova, and B. Gil, Phys. Rev. Lett. 92, 097401 (2004).

${ }^{12}$ W. Lin, Z. Xiang, G. P. Wang, and C. T. Chan, Appl. Phys. Lett. 91, 243113 (2007).

${ }^{13}$ W. Lin and G. P. Wang, Appl. Phys. Lett. 91, 143121 (2007).

${ }^{14}$ A. R. Davoyan, I. V. Shadrivov, A. A. Sukhorukov, and Yu. S. Kivshar, Phys. Rev. A 79, 013820 (2009).

${ }^{15} \mathrm{P}$. Yeh, Optical Waves in Layered Media (Wiley, New York, 1988).

${ }^{16}$ A. R. Davoyan, I. V. Shadrivov, A. A. Sukhorukov, and Yu. S. Kivshar, Opt. Express 16, 3299 (2008). 